\title{
On the concept of individual in ecology and evolution
}

Johan A.J. (Hans) Metz (metz@iiasa.ac.at)

\section{Approved by}

Ulf Dieckmann

Director, Evolution and Ecology Program

February 2015 Institute, its National Member Organizations, or other organizations supporting the work. 


\title{
On the concept of individual in ecology and evolution
}

\author{
J.A.J. Metz ${ }^{1}$ \\ ${ }^{1}$ (= Hans) Institute of Biology and Mathematical Institute \& NCB, Naturalis, \\ Leiden, Netherlands \\ $\&$ \\ Evolution and Ecology Program, IIASA, Laxenburg, Austria \\ j.a.j.metz@biology.leidenuniv.nl
}

Dedicated to Odo Diekmann on the occasion of his 65th birthday.

\begin{abstract}
Part of the art of theory building is to construct effective basic concepts, with a large reach and yet powerful as tools for getting at conclusions. The most basic concept of population biology is that of individual. An appropriately reengineered form of this concept has become the basis for the theories of structured populations and adaptive dynamics. By appropriately delimiting individuals, followed by defining their states as well as their environment, it become possible to construct the general population equations that were introduced and studied by Odo Diekmann and his collaborators. In this essay I argue for taking the properties that led to these successes as the defining characteristics of the concept of individual, delegating the properties classically invoked by philosophers to the secondary role of possible empirical indicators for the presence of those characteristics. The essay starts with putting in place as rule for effective concept engineering that one should go for relations that can be used as basis for deductive structure building rather than for perceived ontological essence. By analysing how we want to use it in the mathematical arguments I then build up a concept of individual, first for use in population dynamical considerations and then for use in evolutionary ones. These two concepts do not coincide, and neither do they on all occasions agree with common intuition-based usage.
\end{abstract}

Key words: concept engineering; individual; conditional independence; structured populations; meso-evolution

Mathematics Subject Classification (2010): 97M60, 92D25, 92D10 


\section{Introduction}

As a birthday present for Odo Diekmann I in this note put the spotlight on a key idea in our many years of collaboration (in the year of his $65^{\text {th }}$ birthday precisely 40 (Diekmann 1978, p. 9)). It is written in the form of a philosophical essay, explicating some intuitions that underlie the mathematical theories of physiologically structured populations and adaptive dynamics. I have chosen this form since I wanted to push those intuitions as far as possible, and possibly even a little further so that the alienating effect may catalyse a change in perspective.

The ideas put forward below derive from a number of different sources. For me it all started with being introduced to the state concept by a popular text on cybernetics by the psychiatrist, W. Ross Ashby (1956). At the time this felt like a revelation. Zadeh and Desoer (1963) and Kalman et al. (1969) later added mathematical depth. Further revelations came from being introduced to the Markov property and renewal points by Feller (1950) and to general age dependent branching processes by Jagers (1975). Finally, I got exposed to the art of building mathematical concepts by Steiner (1964), and to its philosophical and biological counterparts by Russel (1940) and Boche'nski and Menne (1965) respectively Woodger (1937, 1939, 1952).

The essay below deals with a classical problem in the philosophy of biology, the concept of individual. Most philosophical thinking on the topic takes a primarily ontological stand, trying to define individuals in terms of the physical properties of objects that may or may not be classified as such, expressed as single argument predicates. In my perspective, it is far more important how the concept functions as tool in biological arguments. There it matters less what individuals are, than how the presumed individuals occur in relational statements, i.e., more term predicates. An analogy may be found in geometry: mathematicians don't care what points or lines are, as long as they are objects such that through two points "passes" a unique line and two lines "intersect" either in a unique point or not at all, in which case they are called parallel. From this perspective it turns out that the crucial property of individuals is that they satisfy some, restricted, independence conditions. This independence can be engineered by a proper mental construction of (i) the state of an individual, (ii) its environment, on which we then can condition. This construction laid the foundations for the theory of physiologically structured populations and its derived theory of adaptive dynamics, as expounded in i.a. Metz and Diekmann (1986), Diekmann et al. (1990, 1998, 2001, 2003, 2010), Metz and de Roos (1992), Metz et al. (2000) respectively Metz et al. (1992, 1996), Geritz et al. (1998), Diekmann (2004), Durinx et al. (2008), Metz (2012). The earlier ontological perspective then becomes transformed into the pragmatics of constructing proper semantics, tying the mathematical constructs to reality.

\section{Preamble}

\subsection{My philosophical stance}

Concepts are not god-given, but human constructs. In natural discourse their construction happens largely subconsciously, in hindsight referred to as "natural intuition". This intuition comes in the form of "subconscious pre-concepts". Those pre-concepts derive from evolved mechanisms that in interaction with the world around us shape our neural development (seen here as including learning, as when a baby develops its sense of a three dimensional world by experimenting with movement, all the while relating the results of this movement to tactile and visual perception). In scientific discourse the constructions are (sometimes) explicit, although necessarily initially based on natural discourse precursors. Natural discourse concepts, and the scientific ones based on them, often do a good job in matters related 
to our daily functioning, but there is no reason to trust our intuition about further extensions.

Although natural intuition may give us a head start in constructing scientific concepts, the ultimate criterion is not whether these concepts agree with intuition, but whether they do a good job in constructing scientific arguments.

Metarule:

Scientific concepts have to be judged in a forward-looking manner, based on their effectiveness as tools, not backward looking based on their agreement with historical precursors

(the latter variously referred to as "established tradition", "common knowledge", or disguised as "didactical value").

\subsection{Concept engineering}

What matters for letting arguments run smoothly is not so much what things "are" as how they relate to other "things", occurring in the sort of arguments that we are after. A derived rule is that

(1) it usually pays first to consider the structural embedding and only after that to worry about the semantics

(i.e., the interpretation rules in terms of our observational possibilities).

A second derived rule is that

(2) we should be explicit about our scientific goals.

A single concept from natural discourse may have different scientific extensions depending on the context, as defined by what sort of argument we are after.

This goal directedness may seem to clash with rule (1) as scientific goals are often formulated in terms of a semantics. The solution is, as usual, to proceed by trial and error (as in the hypothetico-deductive method).

\subsection{My guiding example}

Mathematics is the science of rigorously delineated conceptual structures. Its basic concepts are structurally defined by their relations, codified in axioms. Axiom systems took form only slowly, guided by their effectiveness for deriving useful results. This usefulness was initially judged largely externally, by semantics connecting the derived results to the observational world, but nowadays more often internally, by

- mapping one mathematical structure into another one,

- interpreting a mathematical structure as abstracting the essentials of some

less abstract ones (with the least abstract structures relating more directly to natural intuition), or, more rigorously,

- deriving the structure through a limit from another one (which is usually more directly interpretable),

- etc.

\subsection{Science: rigorous arguments and fuzzy semantics}

In science, as elsewhere, it is also a good idea to keep structural arguments clean, pertaining to an idealised world, and put any necessary fuzz in the semantics, replacing "=" with "approximates" or "roughly equals", etc. For arguments are settled best by keeping a clear view of the distinction between structure and interpretation, keeping deductive arguments rigorous, and considering the links between theory, intuition and observation separately. 
Most theoretical progress in biology increases the complexity of the structure so as to decrease the fuzz in the semantics.

\subsection{Individuals, history}

In classical logic an individual is anything to which we can assign properties. In classical positivist philosophy the semantics was primarily in terms of spatio-temporal connectedness, and usually also boundedness. With further abstraction, more objects took on the role of individuals (e.g. functions in functional analysis, where in naïve set theory functions are a special type of relations).

The increase in abstraction of our present day reasoning opens the way for a reappraisal of the term/concept. Such a reappraisal is necessarily context dependent, with different concepts of individual being "natural" in different contexts.

\section{Population dynamics}

\subsection{Faithful reproduction and i-states}

In population dynamics theory the essential property characterising individuals is faithful reproduction. To make contact with observational reality this faithfulness should be interpreted in a relaxed manner, in that individuals are allowed over their lifetime to move through different developmental and physiological states, to be called i(nternal)-states, and potentially also to be born with different i-states.

Thus the requirement is that it should be possible to delineate an $i$-state space and a space of environmental conditions such that the mechanism generating the $i$-state dynamics and behavioural output in dependence on the environment reproduces faithfully.

\subsection{Identifying the essentials}

The essential requirement that goes with the state concept is:

Given its present state, the future of an individual is independent of the past.

The essential requirement that goes with the concept of environment is:

Given the environment, individuals behave independently and move independently through their state spaces.

Corollary: Given the environment and given its birth state, an individual is independent of its parent(s).

In view of their importance for all sort of arguments I propose

for population dynamical purposes to take these independences to be the defining properties for the concept of individual.

In this viewpoint the typical properties one tends to associate with the concept of individual like stability and spatiotemporal separation are possible causes of such independence, but should not be made part of the definition. The latter should be based on the structural essentials only.

\subsection{Microscopic versus macroscopic views}

The following two reaction schemes differ microscopically, but lead to the same equations in the large system size limit:

$$
\left(n_{\mathrm{A}}, n_{\mathrm{B}}, n_{C}\right) \stackrel{k \Omega a b}{\longrightarrow}\left(n_{\mathrm{A}}-1, n_{\mathbf{B}}-1, n_{\mathrm{C}}+1\right)
$$




$$
n_{\mathrm{A}} \stackrel{k \Omega a b}{\longrightarrow} n_{\mathrm{A}}-1, \quad n_{\mathrm{B}} \stackrel{k \Omega a b}{\longrightarrow} n_{\mathrm{B}}-1, \quad n_{\mathrm{C}} \stackrel{k \Omega a b}{\longrightarrow} n_{\mathrm{C}}+1
$$

with $a:=n_{\mathrm{A}} / \Omega, b:=n_{\mathrm{B}} / \Omega, \Omega$ the system size and $n_{X}$ the density of $X$-individuals. Conditional on the environment ( $b$ for the A-individuals and $a$ for the B-individuals) in the first scheme A- and B-individuals are dependent in their behaviour, in the second scheme this is not the case. For large systems this microscopic dependence becomes infinitely diluted. The ideas expressed in this note only apply to such large number limits, or to situations where the microscopic and macroscopic environment of individuals coincide (as when organisms interact through some continuous diffusible resource).

Convention about the semantics:

In the stochastic models one should allow for microscopic dependencies that are diluted out when the system is suitably scaled up.

\subsection{Other structures: h-states}

The previous formalisation only captures well-mixed populations. Spatial, social and other external structures can often be included by extending the i-states to $\mathrm{h}$ (eterogeneity)-states, where a h-state consists of an i-state plus some other changeable attribute, like location or position in a social hierarchy, that selects an individual's environmental input from the overall environment, which is then written as a function over the second component of the h-states.

With such an extension the essential independence relations generalise unchanged to populations with external structure.

\subsection{Semantics}

The simplest real objects with a tendency to be approximately (conditionally) independent are bodies, recognisable by their spatio-temporal connectedness and boundedness. Most arguments start from bodies. I will therefore refer to bodies as primary individuals.

Our usual focus on bodies reflects our subconscious pre-concepts. (The spatiotemporal connectedness and boundedness of bodies is only perceptual. On a finer level the molecules making up a body are in a state of constant flux.)

I will call other constructs satisfying the required independence properties generalised individuals, or, when they can be seen as sets of bodies, meta-individuals (in analogy with the term meta-population).

\subsection{An unusual example of generalised individuals}

Imagine equally dividing single celled organisms with size (biomass) as i-state. Assume that cells can divide only when their size $s$ is larger than $s_{1 / 2}:=1 / 2 s_{\max }$. Except when division only occurs at a few fixed sizes, the set of birth states in this imaginary organism is a continuum. It now pays to take the composite of an individual passing through sizes $>s_{1 / 2}$ and its two daughters passing through sizes $<$ $s_{1 / 2}$ as a generalised individual. This way we lessen the dependence between an individual and its parent, leading to simpler mathematical arguments.

\subsection{Meta-populations}

The classical example of meta-individuals are what are usually referred to as the constituting local populations of a meta-population. These meta-individuals are born when an empty patch is settled, and die when the local population goes extinct as a result of demographic stochasticity or a local catastrophe. In the simplest case the istate is the number of bodies in a patch. The environmental condition generally 
consists of at least two variables: density of empty patches and immigration rate. The former co-determines the birth rate, the latter the so-called rescue effect.

For general meta-population models the rescue effect only disappears when extinctions come only from catastrophes.

Part of the semantics that goes with this concept of meta-population is that in the represented real populations

- migration should directly connect relatively large numbers of patches,

- there should be no environmental coupling between patches, other

than

by the effects of migration.

\subsection{Context dependence}

The extent to which objects/constructs are dependent depends on the context. For example, the rescue effect disappears when we concentrate on initial growth of the meta-population. By extrapolation we may expect that some constructs can be considered generalized or meta-individuals in one context, but not in a different one.

\section{Micro-evolution}

\subsection{Definition}

Changes in the genetic constitution of populations are referred to as micro-evolution.

To fit genetics in the earlier framework the genetic constitution of a body should be incorporated as component of its i-state.

\subsection{Population genetics}

Only the simplest ecological scenarios allow decomposition into the standard population genetical equations combined with an ecologically based equation for the density of bodies.

Another option is to consider bodies as communities of genes. (Only in principle, in practice the resulting formalism is horrible.) A single body then has to be counted more than one time, once for each gene copy. (Under random mating the microscopic dependence brought about by their being in a single body disappears in the large system size limit.)

In the latter view the gene copies are the primary individuals, with the phenotype of a gene the map from all possible genetic backgrounds together with which it may find itself in a body, combined with all possible environments in which that body may find itself, to the trait vector measured on the resulting body.

\subsection{Fitness}

In population genetics fitness is traditionally equated to survival till reproduction times average adult offspring production. I will instead call the logarithm of this quantity fitness

as this is the only way to compare models of many different provenances.

This time honoured fitness concept works only for simple ecological scenarios, where the different life phases are both neatly separated and synchronised. Its advantage is that one can introduce fitnesses that vary over space and time. The other advantage of the population genetical simplification of ecology is that it becomes feasible to analyse the effects of complicated genetic architectures.

\section{Meso-evolution}

\subsection{Definition}

I define meso-evolution here as 
evolutionary changes in the values of traits of representative individuals and concomitant patterns of taxonomic diversification.

(In other contexts meso-evolution has been called "long-term" evolution.) Introducing this term frees the term macro-evolution for really large scale changes like anatomical innovations, where one cannot even speak in terms of a fixed set of traits (c.f. Metz 2011).

\subsection{The standard simplification}

Trait evolution tends to depend in interestingly complicated manners on the details of the ecology. Hence we cannot stick to the simple ecological scenarios from population genetics. Yet, some simplifications are needed to argue our way to results. The usual simplifying assumption in meso-evolutionary theory is that

mutants can be considered one at a time.

Unless stated otherwise, all the following statements are predicated on this assumption.

\subsection{ESS theory}

The theory of meso-evolutionary statics is called ESS theory.

Definition:

An Evolutionarily Steady Strategy is a (combination of) resident phenotype(s) that create an environment such that all other phenotypes that can arise through mutation cannot invade.

ESS theory simplifies considerably if we assume that bodies reproduce clonally. Without that assumption we in principle

- have to work with explicit genotype to phenotype maps,

- have to account for genetic structures of any complicatedness.

The natural question then is:

When are the ESSes for models assuming clonally reproducing bodies the same as the ESSes for models that treat bodies as meta-individuals, constructed from genes as the primary individuals?

\subsection{Fitness}

An adequate fitness concept for meso-evolutionary considerations should correctly predict whether or not a mutant can invade, and also the overall speed of such an invasion, for as large as possible a collection of ecological scenarios (Metz et al 1992, Metz 2008).

The basic concept can only be defined for clonally reproducing objects. The best possible extension to the sexual Mendelian case then can be made by mentally constructing clonally reproducing individuals with similar life histories, calculating reproductive output as the average rate of producing kids through the micro- and macro-gametic routes (first add and then divide by 2; see Metz and Leimar 2011, Gyllenberg et al. 2011).

\section{Invasion fitness:}

The fitness $\rho(Y, E)$ of a given type $Y$ in a given ergodic environment $E$ can be defined as the (asymptotic, average) exponential growth rate of a (hypothetical) clone of individuals of that type in that environment.

For mutants the environment $E$ is set by the population dynamics of the resident types. 
(Note the implicit assumption that the resident population dynamics approaches an attractor generating an ergodic environment.) In the population genetical scenario this quantity equals the logarithm of the relative fitness of a heterozygote versus the resident homozygote from the population genetics textbooks.

\subsection{Some results}

Genetically monomorphic ESSes are correctly predicted from the fitness of bodies.

(Heterozygote bodies reproduce effectively clonally by crossing with resident homozygotes.)

Genetic constraints may preclude the extension of this result to genetically polymorphic ESSes.

Example of a genetic constraint: For all environments the fitnesses of phenotypes are similarly ordered, and the fittest type can only be realised by heterozygotes.

Ideal Free assumption:

Mutants can occur that produce any feasible type as heterozygotes in the genetic backgrounds supplied by the resident population.

Proposition: In the IF case also genetically polymorphic ESSes are correctly predicted.

(The proof is only complete for the case of non-fluctuating environments and but a single h-state at birth. See e.g. Bulmer (1994), Metz et al (2008).)

\subsection{Evolutionary individuals}

In view of the preceding considerations, I propose the following concept of

evolutionary individuals:

objects for which one can define a fitness such that ESSes are correctly

predicted.

Bodies may be evolutionary individuals, but this is not always the case (thanks to the evolutionary coupling of bodies by genetic constraints). On the other hand, also other constructs may be considered individuals, like

- family groups (with singles treated as families of size one),

- patches in a structured meta-population,

- pairs in (some) pair approximation calculations for a population

supposedly living on the nodes of a graph

(c.f. Metz \& Gyllenberg 2001, Gyllenberg \& Metz 2001, Parvinen \& Metz 2008, Metz 2008; Matsuda et al. 1992, van Baalen and Rand 1998, van Baalen 2000, Lion and van Baalen 2009).

Note that some objects that cannot be considered population dynamical individuals might yet be evolutionary individuals, since the definition of fitness restricts the considered environments.

\section{Discussion}

\subsection{Updating our intuition}

Some of the above considerations may feel counterintuitive, in particular the consequences of the proposal for the definition of evolutionary individuals. In a genetic polymorphism caused by heterozygote superiority the bodies under 
consideration will not be considered evolutionary individuals. If the viewpoint is changed to a population dynamical one, the bodies are cut loose from the genetic network that keeps them in existence through the ploy of conditioning on their genetic birth state. In the meso-evolutionary context, however, the stress comes to lie on the fact that the homozygote types could never exist were it not for their genetic coupling to heterozygote forebears. Hence evolutionarily they cannot be considered in isolation.

The essence of our naïve intuition about individuals also appears to be that they can be considered as independent entities in agreement with my scientifically motivated proposals, except that our intuition focuses on visible indicators and I on independence per se. (Think of the classical philosophical stress on having a multitude of within individual relations and but a few between individual ones.) In evolutionary arguments we on occasion cannot in our mental constructions get rid of all the connections between individuals, while in population dynamical ones we can. What objects are individuals, thus depends on the imposed context. However, such is the case with all concepts, only usually rather less blatantly. Where our intuition prefers similarity between contexts in our conceptual labelling of objects, I opted for enlarging the reach within each context of the arguments in which the concepts figure.

\subsection{How does all this help mathematical biology?}

The ideas that were described above form part of an informal lore among population modellers. Coming up with some generalized individuals somewhere hidden in the specification of a model can help considerably with its analysis. All I have done in the above essay is making this lore explicit in a slightly facetious manner.

One example of the usefulness of this suite of ideas that I am well familiar with can be found in the introduction of the concept of $R_{\mathrm{m}}$ (the number of immigrants elsewhere produced by a newly established mutant colony in a very large collection of equally coupled patches possibly already populated by some resident types) by Metz and Gyllenberg (2001) and Gyllenberg and Metz (2001). Making the $R_{\mathrm{m}}$ idea explicit sparked of a, small, industry of eco-evolutionary modelling for meta-populations. However, $R_{\mathrm{m}}$ is nothing but the $R_{0}$ (i.e., average life-time offspring production) of an appropriately defined kind of meta-individuals.

Thinking in terms of generalised individuals may also resolve some controversies about the "diversity of $R_{0} \mathrm{~s}$ " appearing in epidemiology. To quote from one of the reviews Claus Rueffler and I received for our contribution to this special issue, " ... it is known that there are many surrogate (biologically irrelevant) indices of $R_{0}$ which can share the threshold property ... ", to which we answered that these quantities are nothing but $R_{0} \mathrm{~s}$ of differently defined meta-individuals. As soon as it is specified what are "births", $R_{0}$ is uniquely determined. However, since the $R_{0}$ of epidemiology is already the $R_{0}$ of a meta-individual (to wit, the population of infective particles in the host body) akin to $R_{\mathrm{m}}$, there actually often is little reason to fix on just that particular quantity. Moreover, the other quantities need not be all that irrelevant. Focussing on a particular type of meta-individual may give one a useful take on a particular epidemiological question. (Notwithstanding the somewhat curt reply we are grateful to that reviewer as her/his remark spurred us to prove that the $R_{0}$-like fitness proxy $Q$ introduced by Metz and Leimar (2011) is unique up to a multiplicative constant if we require that the proxy should be locally sign equivalent to $\ln \left(R_{0}\right)$ and separately affine in the demographic parameters.)

Another example in this area is the seeming clash between the $R_{0}$-concepts for periodic environments introduced by Bacaër and Guernaoui (2006) and by Cushing and Ackleh (2011) (see also Bacaër and Ait Dads, 2012). Basically Bacaër and Guernaoui consider ordinary births, with and extended birth state description also including the phase of the cycle at which individuals are born, whereas Cushing and Ackleh consider generalized individuals of their own making, the construction of 
which, mathematically interesting though it may be, were it only for its unexpectedness, provides little in the way of inspiration for biological thought.

As a final example consider the analysis of the eco-evolutionary model of Shaw and Levin (this issue). In that model one can construct a generalized individual by treating the return to state 1 as if it were a birth. A shortcut to the equilibrium equation is that at equilibrium the $R_{0}$ of these generalized individuals should be 1 . Moreover, at the ESS this $R_{0}$ should be maximal, just as the real $R_{0}$. The fact that the $R_{0}$ of the generalized individuals is separately affine in the parameters considered to be under evolutionary control then makes for the relatively simple analysis of the properties of the ESS.

The concept of evolutionary individuals as such provides little help for mathematical thinking. And even biologists may find it a strange beast. What its introduction does though, is lodge firmly in the mind the idea that if one has derived evolutionary conclusions from ecological considerations it is important to see under precisely which assumptions on the genetical and developmental architecture these conclusions survive the realism check of exposing them to a Mendelian world view.

Although I introduced the concept of evolutionary individuals with my tongue rather close to my cheek, I yet believe it to be useful in that putting it forward brings home that the simple minded approach to evolution concentrating on the performance of bodies can sometimes fail due to the genealogical nexus time and again reconstructing unfit bodies. For population geneticists this is of course nothing new. Yet as a message it at times appears somewhat underappreciated in math biology circles.

Acknowledgements: The present note got of the ground in a workshop on Evolutionary and Ecological Individuals, organised by Philippe Huneman and Minus van Baalen in the context of the multidisciplinary project Ecology and Philosophy: Individuality, Stability \& Ethics, funded by the research program « Ingénierie Ecologique » of the CNRS Institut National Ecologie et Environnement.

\section{$\underline{\text { References }}$}

Ashby WR (1956) Introduction to Cybernetics. Chapmann \& Hall, London

Baalen M van (2000) Pair approximations for different spatial geometries. Pp 359-387 in Dieckmann U, Law R \& Metz JAJ (eds) The Geometry of Ecological Interactions: Simplifying Spatial Complexity. Cambridge Studies in Adaptive Dynamics. Cambridge UP

Baalen M van, Rand DA ((1998) The unit of selection in viscous populations and the evolution of altruism. J Theor Biol 193: 631-648

Bacaër N, Guernaoui S (2006) The epidemic threshold of vector-borne diseases with seasonality: The case of cutaneous leishmaniasis in Chichaoua, Morocco. J Math Biol 53: 421-436

Bacaër N, Ait Dads EH (2012) On the biological interpretation of a definition for the parameter $R_{0}$ in periodic population models. J Math Biol 65: 601-621

Boche'nski IM, Menne A (1965) Grundriss der Logistik. Schöningh, Paderborn

Bulmer M (1994) Theoretical Evolutionary Ecology. Sinauer. Sunderland Ma

Cushing JM, Ackleh AS (2011) A net reproductive number for periodic matrix models. J Biol Dyn 6:166-188

Diekmann O (1978) Over Niet-Lineaire Integraalvergelijkingen en Mathematische Epidemiologie. Mathematisch Centrum, Amsterdam

Diekmann O (2004) A beginner's guide to adaptive dynamics $p$ 47-86 in Mathematical modelling of population dynamics. Banach Center Publications, Volume 63. Institute of Mathematics. Polish Academy of Sciences, Warszawa

Diekmann O, Heesterbeek JAP \& Metz JAJ (1990) On the definition and the computation of the basic reproduction ratio $\mathrm{R}_{0}$ in models for infectious diseases in 
heterogeneous populations. J Math Biol 28: 365-382

Diekmann O, Gyllenberg M, Metz JAJ \& Thieme HR (1998) On the formulation and analysis of general deterministic structured population models I Linear theory. $J$ Math Biol 36: 349-388

Diekmann O, Gyllenberg M, Huang H, Kirkilionis M, Metz JAJ \& Thieme HR (2001) On the Formulation and Analysis of General Deterministic Structured Population Models. II. Nonlinear Theory. J Math Biol 43: 157-189

Diekmann O, Gyllenberg M \& Metz JAJ (2003) Steady state analysis of structured population models. Theor Pop Biol 63: 309-338

Diekmann O, Gyllenberg M, Metz JAJ, Nakaoka S \& de Roos AM (2010) Daphnia revisited: local stability and bifurcation theory for physiologically structured population models explained by way of an example. J Math Biol 61: 277-318

Durinx M, Metz JAJ \& Meszéna G (2008) Adaptive dynamics for physiologically structured models. J Math Biol 56: 673-742. DOI 10.1007/s00285-007-0134-2

Feller W (1950) An Introduction to Probability Theory and its Applications. Wiley, New York.

Geritz SAH, Kisdi É, Meszéna G \& Metz JAJ (1998) Evolutionarily singular strategies and the adaptive growth and branching of the evolutionary tree. Evol Ecol 12: 35-57

Gyllenberg M \& Metz JAJ (2001) On fitness in structured metapopulations. J Math Biol 43: 545-560

Gyllenberg M, Metz JAJ \& Service R (2011) When do optimisation arguments make evolutionary sense? Pp 235-269 in Chalub FACC \& Rodrigues JF (eds) The Mathematics of Darwin's Legacy. Birkhauser, Basel

Jagers P (1975) Branching Processes with Biological Applications. Wiley, London

Kalman RE, Falb PL \& Arbib MA (1969) Topics in Mathematical System Theory. McGraw-Hill, New York

Lion S \& van Baalen M (2009).The evolution of juvenile-adult interactions in populations structured in age and space. Theor Pop Biol 76: 132-145.

Matsuda H, Ogita N, Sasaki A \& Sato K (1992). Statistical mechanics of population. Prog Theor Phys 88: 1035-1049

Metz JAJ (2008) Fitness. Pp. 1599-1612 in Jørgensen SE \& Fath BD (eds) Evolutionary Ecology. Vol. 2 of Encyclopedia of Ecology. Elsevier, Oxford

Metz JAJ (2011) Thoughts on the geometry of meso-evolution: collecting mathematical elements for a post-modern synthesis. Pp 197-234 in Chalub FACC and Rodrigues JF (eds) The Mathematics of Darwin's Legacy. Birkhauser, Basel

Metz JAJ (2012) Adaptive Dynamics. In Hastings A \& Gross LJ (eds) Encyclopedia of Theoretical Ecology. California University Press

Metz JAJ \& Diekmann O (1986) The Dynamics of Physiologically Structured Populations. Lecture Notes in Biomathematics 68. Springer Verlag, Berlin

Metz JAJ \& Gyllenberg M (2001) How should we define fitness in structured metapopulation models? Including an application to the calculation of ES dispersal strategies. Proc Roy Soc B 268: 499-508

Metz JAJ \& Leimar O (2011) A simple fitness proxy for ESS calculations in structured populations with continuous traits, with applications to the evolution of haplo-diploids and genetic dimorphisms. J Biol Dyn 5: 163-190

Metz JAJ \& de Roos AM (1992) The role of physiologically structured population models within a general individual-based modeling perspective. Pp 88-111 in: DL DeAngelis \& LJ Gross (eds) Individual-Based Models and Approaches in Ecology. Routledge, Chapman \& Hall

Metz JAJ, Leimar O (2011) A simple fitness proxy for ESS calculations in structured populations with continuous traits, with applications to the evolution of haplodiploids and genetic dimorphisms. J Biol Dyn 5: 163-190

Metz JAJ, Nisbet RM \& Geritz SAH (1992) How should we define "fitness" for 
general ecological scenarios? TREE 7: 198-202

Metz JAJ, Geritz SAH, Meszéna G, Jacobs FJA \& van Heerwaarden JS (1996) Adaptive dynamics, a geometrical study of the consequences of nearly faithful reproduction. Pp 183-231 in: van Strien SJ \& Verduijn Lunel SM (eds) Stochastic and Spatial Structures of Dynamical Systems. A'dam, North-Holland.

Metz JAJ, Mollison D \& van den Bosch F (2000) The dynamics of invasion waves. Pp 482-512 in Dieckmann U, Law R \& Metz JAJ (eds) The Geometry of Ecological Interactions: Simplifying Spatial Complexity. Cambridge UP.

Metz JAJ, Mylius SD \& Diekmann O (2008) When does evolution optimise? Evol Ecol Res 10: 629-654

Parvinen K \& JAJ Metz (2008) On diploid versus clonal ESSes in metapopulations. Theor Pop Biol 73: 517-528

Rueffler C, Metz JAJ (this issue) Necessary and sufficient conditions for $R_{0}$ to be simple. J Math Biol

Russell B (1940) An Inquiry into Meaning and Truth. Allen \& Unwin, London

Shaw AK, Levin SA (this issue) The evolution of intermittent breeding. $J$ Math Biol

Steiner H-G (1964) Mengen, Abbildungen und Strukturen. Pp 246-292 in Behnke H, Remmert R, Steiner H-G. \& Tietz H (eds) Das Fisher Lexicon Mathematik. Vol I. Fisher Bücherei, Frankfurt am Main

Woodger JH (1937) The Axiomatic Method in Biology. Cambridge University Press

Woodger JH (1939) The Technique of Theory Construction. University of Chicago Press

Woodger JH (1952) Biology and Language. Cambridge University Press

Zadeh LA \& Desoer CA (1963) Linear Systems Theory, the State Space Approach. McGraw-Hill, New York 